\title{
ON A STIELTJES VERSION OF GRONWALL'S INEQUALITY
}

\author{
ANGELO B. MINGARELLI
}

\begin{abstract}
A unified formulation of Gronwall's inequality (GI) for certain Stieltjes integrals is presented. An advantage in using Stieltjes integrals lies in the fact that some of the "continuous" and "discrete" versions of the inequality may be obtained as a consequence of the same theorem.
\end{abstract}

We are concerned here with an extension of the classical GI $[2$, p. 35] to a form which includes most of the Stieltjes formulations of the latter and a more general result of J. V. Herod [3]. We will essentially be following the treatment given in [3] wherein an abstract GI was formulated (Lemma 1 below) generalizing some previous work of Schmaedeke and Sell [10]. In the latter the mean Stieltjes integral (MSI) and the Dushnik or interior integral (DI) (see e.g. [10] and references therein) provided a more general framework than the natural Riemann-Stieltjes integral (RSI), since it allowed the possibility of discontinuous integrands. A sharp version of the GI was given by Jones [4] using RSI. Use of the Lebesgue-Stieltjes integral provided Pandit [8, p. 323] with a tool for the reformulation of GI with the scope of applying the latter to continuous and discrete phenomena (cf. also [4]). F. V. Atkinson [1, p. 455] also gave an extension of GI using the left Cauchy-Stieltjes integral (CSI) (cf. e.g. [9]). Though the result was not sharp in general it provided, as such, a useful tool for the basic study of Volterra-Stieltjes integral equations. Moreover a discrete version could again be obtained by choosing the distribution function appropriately [6], [8].

Our aim is to provide a more general GI (Theorem 1 below) the form of which was prompted by Pachpatte [7].

1. In the sequel all functions will be real-valued, defined on an interval $S \equiv$ $[0, b]$, say, and $\sigma, \mu$ will denote, unless otherwise specified, right-continuous nondecreasing functions on $S$. We will mainly be following [3] in the use of notation. Thus $O B$ will stand for the collection of functions of bounded variation on $S .1$ will denote the constant function whose value is 1 on $S$ whereas $1_{x}$ will be the function whose value is 1 at $x$ and zero elsewhere. Finally $0_{x}=1-1_{x}$ for $x$ in $S$.

As in [3] we let $J$ be a function from $O B$ to the collection of functions from $S \times S$ to the real numbers satisfying the following properties: If each of $f$ and $g$ is

Received by the editors May 22, 1980.

1980 Mathematics Subject Classification. Primary 34A40.

Key words and phrases. Gronwall inequality, Riemann-Stieltjes integral, Cauchy-Stieltjes integral, mean Stieltjes integral, interior integral.

(c) 1981 American Mathematical Society 0002-9939/81/0000-0267/\$02.00 
in $O B$ and $\{x, y, z\}$ is in $S \times S \times S$ then

(1) $J[\alpha f+\beta g](x, y)=\alpha J[f](x, y)+\beta J[g](x, y)$ for real $\alpha, \beta$,

(2) $J[f](x, y)+J[f](y, z)=J[f](x, z)$ if $x\langle y\langle z$ or $x>y\rangle z$,

(3) $J[f](x, y) \geqslant 0$ provided $f(y)>0$ for $x, y, z$ as in (2) above,

(4) if $x$ is in $S$ then each of $J\left[0_{x}\right]\left(x, x^{+}\right)$and $J\left[1_{x}\right]\left(x^{-}, x\right)$ is less than 1 .

We will be assuming further that

(5) for fixed $x$ in $S, J[f](x, \cdot)$ is in $O B$.

If $\sigma, \mu$ are as agreed above, we write $J[f] \equiv J_{\sigma}[f]$ (resp. $J_{\mu}[f]$ ) to show the dependence of $J$ upon $\sigma$ (resp. $\mu$ ). In this case we will suppose that

(6) (i) $J_{\sigma}[f](x, y)=0$ for all $\{x, y\}$ in $S \times S$ if $\sigma$ is identically constant on $S$,

(ii) $J_{\sigma+\mu}[f](x, y)=J_{\sigma}[f](x, y)+J_{\mu}[f](x, y)$ for all $\{x, y\}$ in $S \times S$.

For example it is readily verified that if we think of $J_{0}[f](x, y)$ as a Stieltjes integral of $f$ with respect to $\sigma$ over $[x, y]$ then the MSI and the DI both satisfy (1)-(3) with the former satisfying (4) if we assume in addition that $\sigma(x)-\sigma\left(x^{-}\right)<$ 2 for $x$ in $(0, b]$, and the latter satisfying (4) automatically because of the right-continuity of $\sigma$. Furthermore if we take $J$ to be the left CSI then (1)-(4) are all verified; however the right CSI satisfies (1)-(3) with (4) holding if $\sigma(x)-\sigma\left(x^{-}\right)$ $<1$ for $x$ in $S$. Finally it is immediate that all these Stieltjes integrals necessarily satisfy both (5) and (6) without further assumptions on $\sigma$.

2. LEMMA 1 (Herod [3]). Let $J$ satisfy properties (1)-(4) above. Then there exists a function $m$ from $S \times S$ to the real numbers such that if $f$ is in $O B$ and

$$
f(x)<\varepsilon+J[f](0, x)
$$

for each $x$ in $S$ where $\varepsilon$ is real, then

$$
f(x)<\varepsilon m(0, x)
$$

for each $x$ in $S$.

REMARK 1. The properties of this function $m$ can be found in [3]. In this regard we need only mention that such an $m(0, \cdot)$ is necessarily of bounded variation on $S$ (cf. [3, p. 34, Theorem (iii)]). This lemma is sharp in the sense that equality in (2.1) implies equality in (2.2). Hence a function $J$ which satisfies (1)-(4) "admits" a GI in the sense of Lemma 1. Such a function $J$ will be termed a Gronwall function for simplicity. For the same reason we will call the function $m(0, \cdot)$ whose existence is guaranteed by the lemma a Gronwall majorant.

REMARK 2. In restricting $J$ to a specific Stieltjes integral we note that the hypotheses on the function $f$ may be relaxed somewhat when a GI is sought for that particular integral. For instance, if $J$ is a MSI it suffices only to require that $f$ be right-continuous and, as we saw earlier, that $\sigma(x)-\sigma\left(x^{-}\right)<2$ for $x$ in $(0, b]$ (Kroll and Smith [5, p. 385, Theorem]). On the other hand if $J$ is a left CSI then the right-continuity of $f$ alone is sufficient with no added assumptions on $\sigma$ (cf. [1]). 
TheOREM 1. Let $\sigma, \mu$ be as above and let $J=J_{\sigma}$ and $J=J_{\sigma+\mu}$ be Gronwall functions which satisfy (5) and (6). Let $f$ in $O B$ be nonnegative on $S$ and let $\varepsilon>0$. If, for each $x$ in $S$,

$$
f(x)<\varepsilon+J_{\sigma}[f](0, x)+J_{\sigma}\left[J_{\mu}[f](0, \cdot)\right](0, x),
$$

then

$$
f(x)<\varepsilon\left\{1+J_{0}[n(0, \cdot)](0, x)\right\}
$$

for each $x$ in $S$ where $n(0, \cdot)$ is the Gronwall majorant associated with the Gronwall function $J_{\sigma+\mu}$.

REMARK 3. This result is sharp when $\mu(t) \equiv$ const on $S$ since the right side of (2.4) reduces to the right side of (2.2) in this case.

We obtain the result in [7] by letting $\sigma, \mu$ both be nonnegative continuously differentiable functions on $S$ and defining $J$ by $J_{\sigma}[f](0, x)=\int_{0}^{x} f \sigma^{\prime} d s$, etc.

3. Proof of Theorem 1. Let $v(x)$ denote the right side of (2.3). Since $f(x)<v(x)$

$$
v(x)<\varepsilon+J_{\sigma}[v](0, x)+J_{\sigma}\left[J_{\mu}[v](0, \cdot)\right](0, x)
$$

because of the monotone property of $J$ (cf. (3)),

$$
\begin{aligned}
& =\varepsilon+J_{\sigma}\left[v+J_{\mu}[v](0, \cdot)\right](0, x) \\
& =\varepsilon+J_{\sigma}[V](0, x)
\end{aligned}
$$

if we define $V(x)$ to be the function in (3.1) within the outer square brackets. Note that $v(x)$ (hence $V(x)$ ) is of bounded variation on $S$, because of the hypotheses (5)-(6), and nonnegative. So $V(t)>v(t)$. This, along with (3.2), implies that

$$
\begin{aligned}
V(x) & =v(x)+J_{\mu}[v](0, x) \\
& <\varepsilon+J_{\sigma}[V](0, x)+J_{\mu}[V](0, x) \\
& =\varepsilon+J_{\sigma+\mu}[V](0, x) .
\end{aligned}
$$

The hypotheses on $J_{\sigma+\mu}$ along with Lemma 1 imply the existence of a Gronwall majorant $n(0, \cdot)$ such that

$$
V(x)<\varepsilon n(0, x)
$$

for each $x$ in $S$. Now inserting (3.4) into (3.2) and making use of (1) we find $v(x)<\varepsilon+\varepsilon J_{\sigma}[n(0, \cdot)](0, x)$. The conclusion now follows.

The author expresses his thanks to the referee for some useful comments and to Professor G. R. Sell for providing some additional references.

\section{REFERENCES}

1. F. V. Atkinson, Discrete and continuous boundary problems, Academic Press, New York, 1964.

2. R. Bellman, Stability theory of differential equations, McGraw-Hill, New York, 1955.

3. J. V. Herod, A Gronwall inequality for linear Stieltjes integrals, Proc. Amer. Math. Soc. 23 (1969), 34-36.

4. G. S. Jones, Fundamental inequalities for discrete and discontimuous functional equations, SIAM J. Appl. Math. 12 (1964), 43-57.

5. J. R. Kroll and K. P. Smith, An eigenvalue problem for the Stieltjes mean sigma-integral related to Gronwall's inequality, Proc. Amer. Math. Soc. 33 (1972), 384-388. 
6. A. B. Mingarelli, Volterra-Stieltjes integral equations and generalized differential expressions, Ph.D. Dissertation, University of Toronto, 1979.

7. B. B. Pachpatte, A note on Gronwall-Bellman inequality, J. Math. Anal. Appl. 44 (1973), 758-762.

8. G. B. Pandit, On Stieltjes-Volterra integral equations, Bull. Austral. Math. Soc. 18 (1978), 321-344.

9. G. B. Price, Cauchy-Stieltjes and Riemann-Stieltjes integrals, Bull. Amer. Math. Soc. 49 (1943), 625-630.

10. W. W. Schmaedeke and G. R. Sell, The Gronwall inequality for modified Stieltjes integrals, Proc. Amer. Math. Soc. 19 (1968), 1217-1222.

Department of Mathematics, University of Ottawa, Ottawa, Ontario, Canada Kin 9 B4 